\title{
Avaliação das relações do ambiente versus a prática de atividade física em municípios de pequeno porte
}

As taxas de desenvolvimento das últimas décadas mostram o transporte, que faz aumentar o consumo de energias não renováveis, como elemento estratégico. Este consumo contribui fortemente para a elevação de níveis de emissão de dióxido de carbono (CO2), um prejuízo para o ambiente. A substituição do transporte motorizado por deslocamentos ativos contribuem para a melhora da saúde da população, para a redução de custos com tratamentos em saúde e são potenciais para redução de emissões de gases poluentes. 0 objetivo deste estudo foi avaliar a relação entre atividade física (AF) e ambiente em três municípios de pequeno no Vale do Taquari, Rio Grande do Sul. Fizeram parte da análise 109 idosos em Roca Sales, 253 adultos em Muçum e 203 adultos em Lajeado. Como instrumentos foram utilizados o questionário internacional de atividade física (IPAO) versão curta para adultos e versão longa adaptada para idosos e a Neighborhood Environment Walkability Scale (NEWS), versão adaptada. Os resultados apresentaram associação positiva significativa para melhores níveis de AF, sendo que os idosos e a Neighborhood Environment Walkability Scale (NEWS), versão adaptada. Os resultados apresentaram associação positiva significativa para melhores níveis de AF, sendo que os
escores de qualidade de calçadas para idosos $(p=0,030)$ de Roca Sales; de acessibilidade e conveniência $(p=0,040)$, apoio social $(p=0,014)$, segurança no trânsito $(p=0,015)$ e animal de estimação $(p=0,028)$ dentre os adultos de Muçum e apenas de apoio social $(p=0,046)$ e segurança geral $(p=0,035)$ para os adultos de Lajeado. Por meio dos resultados, pode-se inferir que maiores densidades populacionais podem favorecer um deslocamento ativo por conta de maior facilidade de acesso a pontos de interesse, assim como por melhores níveis de segurança e melhor qualidade de calçadas. No entanto, maiores densidades também concentram mais veículos individuais e menos segurança. Cidades menores tem no menor acesso um problema para o transporte ativo, mas conferem melhores condições nos demais itens, trazendo melhores possibilidades para a substituição do transporte motorizado pelo ativo fisicamente, potencializando a redução de emissões. Entende-se que estudos individualizados, levando em consideração as especificidades de cada município devam ser realizados por não ficarem evidentes similaridades que potencializem o deslocamento ativo.

Palavras-chave: Ambiente; Emissão de gases; Saúde; Deslocamento ativo.

\section{Assessment of environmental relationships versus physical activity in small cities}

\begin{abstract}
The development rates of the last decades have shown transportation, which increases the consumption of non-renewable energy, as a strategic element. This consumption contributes strongly to the in res of the population's health, reduction of health treatment costs and its potential to reduce pollutant gas emissions. The objective of this study was to evaluate the relationship between of the population's health, reduction of health treatment costs and its potential to reduce pollutant gas emissions. The objective of this study was to evaluate the relationship between
physical activity (PA) and the environment in three small cities in Vale do Taquari, Rio Grande do Sul. The analysis included 109 elderly people in Roca Sales, 253 adults in Muçum and 203 physical activity (PA) and the environment in three small cities in Vale do Taquari, Rio Grande do Sul. The analysis included 109 elderly people in Roca Sales, 253 adults in Muçum and 203
adults in Lajeado. As instruments were used the international physical activity questionnaire (IPAQ), short version for adults and long version adapted for elders and an adapted version of the Neighborhood Environment Walkability Scale (NEWS). The results showed a significant positive association for better PA levels, with the sidewalk quality scores for the elderly ( $p=0.030$ ) in Roca Sales; accessibility and convenience $(p=0.040)$, social support $(p=0.014)$, traffic safety $(p=0.015)$ and pets $(p=0.028)$ among adults in Muçum and only social support ( $p=0.046)$ and general safety $(\mathrm{p}=0.035)$ for adults in Lajeado. Through the results, it can be inferred that higher population densities may favour an active mobility due to easier access to points of interest as well as better levels of safety and better quality of sidewalks. However, higher densities also concentrate more individual vehicles and less safety. Smaller cities have less access to problem for active mobility, but they provide better conditions for other factors, bringing better possibilities to substitute motorized transport for physically active transport, enhancing the reduction of emissions. It is understood that individualized studies, taking into account the speificites of each city, should be caried out because there are no evident similaites that

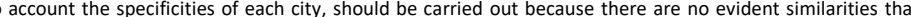
enhance active mobility.
\end{abstract}

Keywords: Environment; Emission of gases; Health; Active mobility.

Topic: Planejamento, Gestão e Políticas Públicas Ambientais

Reviewed anonymously in the process of blind peer.
Received: 05/05/2020 Approved: 07/06/2020
Leonardo de Ross Rosa (iD)

Universidade do Vale do Taquari, Brasil http://lattes.cnpq.br/9997046654327810 http://orcid.org/0000-0003-1448-4758

Idrrosa@univates.br

Ricardo Bronca

Universidade do Vale do Taquari, Brasil http://lattes.cnpq.br/7089174468372682

http://orcid.org/0000-0002-4670-1254

rbronca@universo.univates.br

Rodolfo Pavi (iD

Universidade do Vale do Taquari, Brasil

http://lattes.cnpq.br/6161719540904823

http://orcid.org/0000-0002-5171-9803

rpavi@universo.univates.br

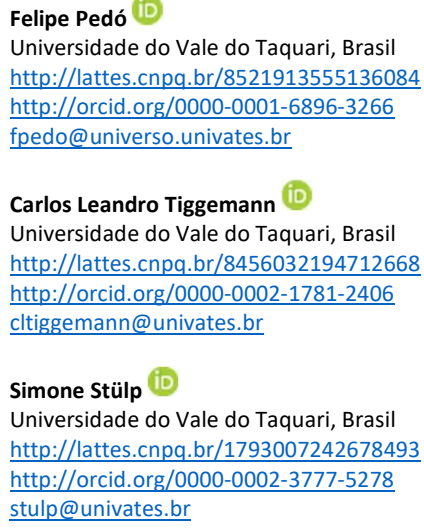

Universidade do Vale do Taquari, Brasil http://lattes.cnpq.br/8521913555136084 http://orcid.org/0000-0001-6896-3266 fpedo@universo.univates.br

Carlos Leandro Tiggemann (iD)

Universidade do Vale do Taquari, Brasil http://lattes.cnpq.br/8456032194712668 http://orcid.org/0000-0002-1781-2406 cltiggemann@univates.br

Simone Stülp (iD

Universidade do Vale do Taquari, Brasil http://lattes.cnpq.br/1793007242678493

http://orcid.org/0000-0002-3777-5278

stulp@univates.br

\section{Referencing this:}

ROSA, L. R.; BRONCA, R.; PAVI, R. PEDÓ, F.; TIGGEMANN, C. L.; STÜLP S.. Avaliação das relações do ambiente versus a prática de atividade física em municípios de pequeno porte. Revista Ibero-Americana de Ciências Ambientais, v.11, n.4, p.450-462, 2020. DOI: http://doi.org/10.6008/CBPC2179-6858.2020.004.0038 


\section{INTRODUÇÃO}

As taxas de desenvolvimento das últimas décadas fizeram aumentar o consumo de energias não renováveis como carvão, petróleo e gás, fato que aponta para uma relação direta entre crescimento econômico e consumo de energia. Este, contribui fortemente para a elevação de níveis de emissão de dióxido de carbono $\left(\mathrm{CO}_{2}\right)$, considerado o principal responsável pelo aquecimento global (DANISH et al., 2017; PÉLLICO NETTO et al., 2008).

O crescimento econômico tem no transporte um elemento estratégico. É componente de destaque no planejamento urbano, não apenas por conectar os espaços, mas também por seu influxo ambiental relevante. Carvalho (2011) afirma que para a população em geral, a poluição se manifesta claramente a partir da descarga de veículos, principalmente os que emitem a fumaça escura, com material particulado. No entanto, apesar de as emissões menos expressivas visualmente não receberem a mesma atenção, estão agregadas na configuração de um importante problema ambiental a partir da queima de combustíveis fósseis.

A produção de veículos automotores com queima destes combustíveis, assim como o volume de atividades para o desenvolvimento e a necessidade dos mesmos, aumentou de maneira desproporcional em relação a alternativas viáveis ambientalmente. Neste contexto, é essencial recuperar que o Brasil tem $80 \%$ de sua população nos perímetros urbanos, valor superior à estimativa mundial para os anos de 2050 (BAKICI et al., 2012). Assim, os espaços urbanos, onde se concentram estes atores têm, naturalmente, aumentadas as concentrações de gases poluentes emitidos.

A emissão de gases de efeito estufa dos transportes, corresponde a $25 \%$ de todas as emissões globais (ANDRADE et al., 2019). No Brasil o setor de transportes é responsável por cerca de $9 \%$ das emissões de $\mathrm{CO}_{2}$ (CNT, 2009). Carvalho (2011) aponta que o transporte rodoviário por meio de ônibus corresponde a 60\% dos deslocamentos urbanos e estes são responsáveis por 7\% das emissões. De outra parte, veículos comerciais leves e automóveis contribuem com menos de $30 \%$ das viagens, mas correspondem a mais da metade das emissões de $\mathrm{CO}_{2}$. Em pesquisa coordenada pelo IPEA Instituto de Pesquisa Econômica Aplicada (IPEA), o transporte individual corresponde a aproximadamente 35\% das viagens motorizadas, sendo a elas atribuídas quase $60 \%$ das emissões de $\mathrm{CO}_{2}$ nos centros urbanos. No mundo, até 2050 , a expectativa é de que o consumo desta energia dobre de volume, apesar do empenho e compromisso dos países na criação de políticas ambientais para a redução das emissões (ZHANG et al., 2016; ANDRADE et al., 2019). Complementando, a eficiência ambiental dos municípios brasileiros é baixa, sendo ainda piores nos municípios de pequeno porte em relação aos grandes centros (SOARES et al., 2019). Frente a isso, a simples alteração do veículo individual pelo coletivo já teria reflexos positivos para o meio ambiente (CARVALHO, 2011; ANDRADE et al., 2019).

De outra parte, a substituição de transporte motorizado por deslocamentos a pé ou mesmo de bicicleta, além de contribuir para a melhora da saúde da população no que diz respeito aos níveis de atividade física (AF), reduz consideravelmente as emissões que impactarão também na melhoria da saúde geral, haja vista que os veículos individuais são os responsáveis pelas viagens mais curtas, ou seja, são passíveis de 
substituição por caminhadas ou viagens de bicicleta. Leite et al. (2012) apontam que em Copenhague, na Dinamarca, considerada uma das cidades mais sustentáveis do mundo, essa troca faz parte da realidade de $37 \%$ da população. Ademais, todo o transporte público teve substituídos os combustíveis fósseis por energia a bateria. É relevante lembrar que no continente europeu, principalmente nas cidades de altas densidades demográficas, as bicicletas são a forma preferida de deslocamento da população (LEITE et al., 2012).

O design urbano influencia a prática de atividades físicas por adultos (SALLIS et al., 2016). Este incremento nos níveis de AF da população a partir de medidas baseadas no desenvolvimento urbano, certamente traria uma menor emissão de gases pela redução do trânsito de veículos automotores. Da mesma forma, uma reestruturação das cidades, também sobre as bases do conceito das cidades inteligentes, com atenção para o florestamento e o reflorestamento - participantes da neutralização do $\mathrm{CO}_{2}$ - no planejamento urbano, podem contribuir sobremaneira para a redução dos impactos do $\mathrm{CO}_{2}$ na atmosfera (PÉLLICO NETTO et al., 2008).

O planejamento urbano é foco de estudos que buscam por uma maior eficiência, redução de impactos ambientais e melhoria da qualidade de vida da população (HANDY et al., 2002). Frank et al. (2005) entendem que o homem disputa espaço com os carros e quanto mais veículos temos, menores as oportunidades de caminharmos, o que indiretamente conduz ao sedentarismo. Autores como Brownson et al. (2009) e Hino et al. (2010), relatam que a relação da AF com o ambiente deve considerar o comportamento de viagem. $\mathrm{O}$ indivíduo pode fazer uma caminhada com fim em si, ou realizar um deslocamento de bicicleta com fim no seu destino. Assim, estará realizando atividade física sem que este seja o seu objetivo específico, que é chegar ao destino.

Os modelos ecológicos de análise direcionam a atenção para os fatores ambientais e de políticas públicas que podem ser o princípio dos problemas de estilos de vida sedentários. Apesar da prática de AF ser uma escolha pessoal, a discussão na coletividade, a urgência do momento, representada pelos números alarmantes que relacionam AF e saúde, sugere ações independentes do interesse pessoal. Melhores condições para o fomento do transporte ativo vem ao encontro das necessidades atuais, tanto por conta da saúde da população, que deriva em custo gerado para o Estado (WEN et al., 2012), quanto na relação da redução de veículos com o ambiente.

Ademais, os fatores psicológicos e sociais são provavelmente mais importantes para andar do que para dirigir, assim como a criminalidade e a paisagem estética, que não influenciam no comportamento de dirigir, mas interferem no deslocamento ativo (HANDY et al., 2002; HINO et al., 2010; FRANK et al., 2005). Da mesma forma, Handy et al. (2002) concordam que caminhar e andar de bicicleta estão mais relacionados à prática de AF e ao recreativo do que como forma de deslocamento. Concluem também que uma maior e melhor orientação de pedestres e um ambiente construído favorável, com um uso diversificado da terra e uma boa conectividade de ruas, podem aumentar, tanto a viabilidade, quanto a atratividade de caminhada e ciclismo. Fatores como a beleza e a localização geográfica são estímulos para a prática de AF e para deslocamentos a pé.

Isso sugere atenção para a criação ou a reformulação de pequenos centros urbanos que possibilitem 
uma aproximação dos pontos de necessidade dos indivíduos. Pessoas que vivem em áreas mais densas, com ruas bem conectadas e uso do solo mais diversificado, tendem a ser mais fisicamente ativas (FRANK et al., 2006; OWEN et al., 2007; DYCK et al., 2010). Frank et al. (2005) reforçam que quanto maior o mix do uso da terra, maior a conectividade e mais possibilidades de deslocamento serem realizados a pé. De outra parte, cidades menores tendem a uma menor densidade, menor oferta de serviços. Comumente as cidades conservam características que não estimulam a prática de deslocamentos não motorizados por conta das distâncias, representadas pela pequena diversidade do uso da terra, e pela segurança, entre outros (FRANK et al., 2006; OWEN et al., 2007; DYCK et al., 2010; FRANK et al., 2005). A formatação mais comum é a de centros que concentram as mesmas atividades, indo de encontro ao conceito de mix do uso de terra. A mudança nesta realidade passa por uma nova organização dos centros urbanos.

Os estudos envolvendo ambiente e AF, em maioria, são estruturados a partir de países desenvolvidos e de alta renda. Outras publicações ainda têm como cerne nações em desenvolvimento, latino-americanas como Colômbia e Brasil, mas mesmo assim, tendo como foco principal grandes centros urbanos, cujas consideráveis densidades demográficas e maior conectividade apresentam-se determinantes na relação entre AF e ambiente (ARANGO et al., 2013; FLORINDO et al., 2012; HINO et al., 2010). O objeto deste estudo foi analisar a relação da AF com a percepção do ambiente construído em três municípios gaúchos de pequeno porte situados no Vale do Taquari, Rio Grande do Sul, Brasil.

\section{METODOLOGIA}

Trata-se de um estudo transversal, comparativo e descritivo realizado nas cidades de Roca Sales, Muçum e Lajeado, todas de pequeno porte e localizadas no Vale do Taquari, região leste do Rio Grande do Sul, Brasil. Foram mensurados níveis de AF e escores de percepção do ambiente e observada sua associação em cada um dos municípios. Em Roca Sales, o estudo ocorreu com a população idosa, já em Muçum e Lajeado a população estudada foi de adultos. A opção pelas amostras se deu por conveniência. Para melhor descrição e compreensão das características das cidades envolvidas, optou-se pela distribuição dos dados em tabela.

Tabela 1: Dados das cidades de Roca Sales, Muçum e Lajeado. *

\begin{tabular}{|c|c|c|c|}
\hline & Roca Sales & Muçum & Lajeado \\
\hline Amostra & 109 idosos & 253 adultos & 203 adultos \\
\hline $\begin{array}{l}\text { Distância da capital, Porto } \\
\text { Alegre em km }\end{array}$ & 143,55 & 114,47 & 114,00 \\
\hline Área em km² & 208.630 & 111.234 & 91.591 \\
\hline População em habitantes** & 11.101 & 4.954 & 84.014 \\
\hline $\begin{array}{l}\text { Densidade demográfica em } \\
\text { hab } / \mathrm{km}^{2}\end{array}$ & 49,29 & 43,20 & 793,07 \\
\hline População urbana em \% & 63,60 & 82,12 & 99,62 \\
\hline $\begin{array}{l}\text { População foco do estudo: } \\
\text { idosos ou adultos residentes em } \\
\text { área urbana*** }\end{array}$ & 1.169 & 2.148 & 43.997 \\
\hline $\begin{array}{l}\text { Enquadramento por número de } \\
\text { habitantes }\end{array}$ & pequeno porte & pequeno porte & pequeno porte \\
\hline IDH & 0,729 & 0,746 & 0,778 \\
\hline $\begin{array}{l}\% \text { Arborização em áreas } \\
\text { públicas }\end{array}$ & 88,80 & 97,20 & 92,70 \\
\hline \% Urbanização de vias públicas & 35,10 & 51,60 & 44,10 \\
\hline colonização predominante & alemã / italiana & italiana & alemã \\
\hline
\end{tabular}




\begin{tabular}{|l|l|l|l|}
\hline $\begin{array}{l}\text { Presença de espaços públicos } \\
\text { para AF }\end{array}$ & não & sim \\
\hline $\begin{array}{l}\text { Características geográficas } \\
\text { gerais em relação a ambientes } \\
\text { naturais }\end{array}$ & $\begin{array}{l}\text { possui espaços naturais como } \\
\text { montanhas, rios e riachos em } \\
\text { torno da região urbana }\end{array}$ & $\begin{array}{l}\text { possui espaços naturais como } \\
\text { montanhas, rios e riachos em } \\
\text { torno da região urbana }\end{array}$ & $\begin{array}{l}\text { possui urbana } \\
\text { extensa com } \\
\text { fragmentos de vegetação }\end{array}$ \\
\hline
\end{tabular}

*Dados do Censo 2010.

**A população estimada para 2019.

*** População foco: idosos em Roca Sales e adultos em Muçum e Lajeado.

\section{População e amostra}

As cidades foram escolhidas por acessibilidade dos pesquisadores. As amostras foram calculadas a partir de um percentual dos residentes em área urbana dos municípios. Em Roca Sales foram amostras 9,32\% da população idosa residente na zona urbana do município. Em Muçum a amostra chegou a 11,77\% dos adultos residentes em área urbana do município. Em Lajeado, atingiu-se um percentual de 0,46\% dos residentes adultos da área urbana da cidade. Foram critérios de inclusão: residir a pelo menos seis meses na cidade; não possuir nenhum déficit cognitivo; não possuir nenhum impedimento para a prática de atividades físicas. Foram excluídos os sujeitos que não responderam os questionários em sua totalidade. O TCLE foi lido e posteriormente assinado pelos participantes da pesquisa. No caso dos formulários digitalizados, o termo foi encaminhado anexo ao instrumento para a leitura.

Todos os participantes foram voluntários e convidados a partir de abordagem pessoal, no caso dos sujeitos (idosos) da cidade de Roca Sales em virtude da não familiarização com tecnologias digitais. Já em Muçum, a resposta aos instrumentos se deu por meio digital com os mesmos instrumentos adaptados para formulários do Google. O link de pesquisa foi divulgado por mídias digitais. O estudo foi aprovado pelo Comitê de Ética e Pesquisa sob número do parecer: 2.547.603.

\section{Materiais}

Nos estudos, para avaliação da percepção do ambiente, foi utilizada a versão adaptada por Florindo et al. (2012) da escala Neighborhood Environmental Walkability Scale (NEWS), traduzida por Malavasi et al. (2007). Na adaptação foram somadas questões da escala de apoio social para a prática de atividade física desenvolvida e validada por Reis et al. (2011). A adaptação foi estruturada para se adequar à realidade brasileira, por conta das características dos países desenvolvidos, onde a NEWS é amplamente utilizada. A versão final do instrumento conta com 38 questões, a saber: ambiente construído/estruturas ambientais para a prática (18 questões); calçadas (duas questões); áreas verdes (duas questões); topografia das ruas (uma questão); poluição ambiental (três questões); segurança no trânsito (três questões); segurança geral (três questões); apoio social (três questões); clima (uma questão); animal doméstico/cachorro (duas questões). As respostas geram um escore numérico para cada âmbito de análise. A primeira parte da escala (questões 1 até 18) foi estruturada para que os indivíduos respondessem quanto tempo levariam caminhando para chegar das suas residências até pontos de interesse, sendo considerado próximos os destinos com tempo menor do que dez minutos de caminhada e distantes os destinos onde são necessários dez ou mais minutos de caminhada. A mesma gera um escore de 1 a 18 . Nas questões 19 a 25, a abordagem 
trata das condições sobre o ambiente próximo de suas casas, como calçadas, ruas, arborização, lixo e esgoto. Os respondentes são orientados para considerar como perto de suas residências os locais aos quais eles conseguem chegar em até 10 minutos caminhando. As demais questões foram compostas por opções de respostas categorizadas de forma dicotômica (sim ou não) ou politômica (ruim, regular ou bom). Os escores são estabelecidos da seguinte maneira: Escore de acessibilidade a conveniências (questões 1 a 18) varia de 1 a 18; os Escores de segurança no trânsito, segurança geral, apoio social e poluição geral, variam de 0 a 3 , sendo zero ruim e 3 bom; o Escore de animal de estimação varia de 0 a 2, sendo 0 ruim e 2 bom. Os escores referentes à topografia, que varia de 0 a 3, e de clima, que varia de 0 a 1, são invertidos ou seja, quanto mais próximo de zero, melhor.

Para a avaliação do nível de AF dos adultos, foram utilizadas duas versões do questionário internacional de atividade física International Physical Activity Questionnaire (IPAQ). No estudo em Muçum e em Lajeado, foi utilizada a versão curta validada no Brasil por Matsudo et al. (2001). Já para a avaliação dos idosos na cidade de Roca Sales, foi utilizada a versão longa do mesmo instrumento adequada para esta faixaetária de 60 anos ou mais. A mesma foi validada por Benedetti et al. (2004). A versão para idosos é composta por 15 perguntas distribuídas em 5 domínios: atividades físicas no trabalho, atividades físicas como meio de transporte, atividades físicas em casa: tarefas domésticas e família, atividades físicas de recreação, esporte, exercício físico e de lazer e tempo gasto sentado. Ambas as versões aferem o tempo gasto em diferentes atividades físicas, com um tempo mínimo de dez minutos no período de uma semana. $\mathrm{O}$ instrumento classifica os níveis de AF em sedentário ou baixo, ativo moderado ou ainda ativo intenso.

Para os adultos das cidades de Muçum e Lajeado foi disponibilizado link de pesquisa para o qual os instrumentos foram adaptados. O mesmo foi divulgado em mídias sociais. Para os idosos de Roca Sales os instrumentos de pesquisa foram impressos e a coleta se deu através de abordagem aos grupos de convivência existentes no município.

\section{Análise de dados}

A análise dos dados foi realizada por meio de estatística descritiva (média, desvio padrão e distribuição de frequência). A comparação das variáveis entre os diferentes grupos, inativos, ativos moderados e ativos intensos, foi realizada por meio do teste $U$ de Mann-Whitney para amostras independentes, sendo adotado um nível de significância de $p<0,05$. Todos os procedimentos foram constituídos no software SPSS versão 20.0 .

\section{RESULTADOS}

A partir da aplicação dos instrumentos nas duas populações nas cidades de Roca Sales, Muçum e Lajeado, foram encontrados os resultados que seguem. Em relação aos dados da cidade de Roca Sales, foram sujeitos de pesquisa 109 indivíduos, homens e mulheres acima de 60 anos. Destes, o percentual de fisicamente ativos, somando moderados e intensos, chega a $81,65 \%$ para apenas $18,35 \%$ de inativos. Observando a tabela 2 , apenas o escore que diz respeito à qualidade das calçadas apresentou associação 
significativa positiva $(p<0,030)$ em prol dos ativos fisicamente.

Tabela 2: Resultados das médias, desvio padrão e nível de significância com idosos em Roca Sales.

\begin{tabular}{l|llll}
\hline \multirow{2}{*}{ Domínio } & \multicolumn{1}{l}{ Total ( $\mathrm{n}$ - 109) } & Ativos ( $\mathrm{n}$ - 89) & Inativos (no 20) \\
\cline { 2 - 5 } & Média dp & Média dp & Média dp & $\mathrm{p}$ \\
\hline Acessibilidade & $7,41 \pm 3,62$ & $7,56 \pm 3,602$ & $6,75 \pm 3,74$ & 0,330 \\
Calçadas (0 a 1) & $0,61 \pm 0,489$ & $0,66 \pm 0,475$ & $0,40 \pm 0,503$ & $\mathbf{0 , 0 3 0}$ \\
Áreas verdes (0 a 1) & $0,72 \pm 0,453$ & $0,72 \pm 0,452$ & $0,70 \pm 0,470$ & 0,865 \\
Topografia (0 a 1) & $0,55 \pm 0,500$ & $0,54 \pm 0,501$ & $0,60 \pm 0,503$ & 0,624 \\
Poluição geral* (3 a 0) & $0,37 \pm 0,676$ & $0,36 \pm 0,711$ & $0,40 \pm 0,503$ & 0,311 \\
Segur. de trânsito (0 a 3) & $1,83 \pm 0,776$ & $1,81 \pm 0,782$ & $1,95 \pm 0,759$ & 0,537 \\
Segurança geral (0 a 3) & $1,85 \pm 0,705$ & $1,89 \pm 0,730$ & $1,70 \pm 0,571$ & 0,213 \\
Apoio Social (0 a 3) & $0,61 \pm 0,792$ & $0,66 \pm 0,825$ & $0,40 \pm 0,598$ & 0,255 \\
Clima* (1 a 0) & $0,73 \pm 0,444$ & $0,73 \pm 0,446$ & $0,75 \pm 0,444$ & 0,858 \\
Animal ** & $0,16 \pm 0,371$ & $0,17 \pm 0,383$ & $0,10 \pm 0,316$ & 0,526
\end{tabular}

*Somatório invertido: quanto mais próximo de 'zero' melhor o resultado para a atividade física.

**Amostra considerada foi das pessoas que tinham animais de estimação.

Em recorte dos cenários de prática física dentre os ativos, é absolutamente relevante o contexto doméstico, onde $62,92 \%$ dos ativos fisicamente acumula mais de $50 \%$ do total de seu tempo de AF. Na cidade de Muçum, da amostra de 253 adultos, 51\% dos munícipes foram considerados sedentários e 49\% fisicamente ativos, sendo destes: $31,6 \%$ fisicamente ativos moderados e 17,4\% fisicamente ativos intensos. Quando comparados os sujeitos ativos e sedentários (Tabela 3), constatou-se diferenças significativas em favor dos ativos fisicamente nos escores de acessibilidade e conveniência $(p<0,040)$, de apoio social $(p<0,014)$, de segurança no trânsito $(p<0,015)$ e o escore animal de estimação $(p<0,028)$.

Tabela 3: Resultados das médias, desvio padrão e nível de significância com adultos em Muçum.

\begin{tabular}{|c|c|c|c|c|}
\hline \multirow[t]{2}{*}{ Domínio } & \multirow{2}{*}{$\begin{array}{l}\text { Total (no 253) } \\
\text { Média dp }\end{array}$} & \multirow{2}{*}{$\begin{array}{l}\text { Ativos (no 124) } \\
\text { Média dp }\end{array}$} & \multicolumn{2}{|c|}{ Inativos (no 129) } \\
\hline & & & Média dp & $p$ \\
\hline Acessibilidade & $8,25 \pm 3,53$ & $8,71 \pm 3,69$ & $7,81 \pm 3,34$ & 0,040 \\
\hline Calçadas (0 a 1) & $0,70 \pm 0,461$ & $0,74 \pm 0,439$ & $0,65 \pm 0,478$ & 0,117 \\
\hline Áreas verdes ( 0 a 1 ) & $0,92 \pm 0,270$ & $0,90 \pm 0,308$ & $0,95 \pm 0,227$ & 0,137 \\
\hline Topografia (0 a 1) & $0,81 \pm 0,397$ & $0,77 \pm 0,420$ & $0,84 \pm 0,371$ & 0,206 \\
\hline Poluição geral* (3 a 0) & $0,21 \pm 0,445$ & $0,24 \pm 0,500$ & $0,18 \pm 0,384$ & 0,457 \\
\hline Segurança de trânsito ( 0 a 3) & $1,52 \pm 0,978$ & $1,67 \pm 0,926$ & $1,38 \pm 1,009$ & 0,015 \\
\hline Segurança geral (0 a 3) & $2,60 \pm 0,668$ & $2,61 \pm 0,647$ & $2,60 \pm 0,691$ & 0,964 \\
\hline Apoio Social (0 a 3) & $0,78 \pm 0,840$ & $0,91 \pm 0,865$ & $0,65 \pm 0,797$ & 0,014 \\
\hline Clima* (1 a 0$)$ & $0,56 \pm 0,498$ & $0,57 \pm 0,497$ & $0,54 \pm 0,500$ & 0,632 \\
\hline Animal $* *$ & $0,76 \pm 0,817$ & $0,51 \pm 0,503$ & $0,34 \pm 0,476$ & 0,028 \\
\hline
\end{tabular}

*Somatório invertido: quanto mais próximo de 'zero' melhor o resultado para a atividade física.

**Amostra considerada foi das pessoas que tinham animais de estimação.

Tabela 4: Resultados das médias, desvio padrão e nível de significância com adultos em Lajeado.

\begin{tabular}{l|llll}
\hline Variáveis & \multicolumn{2}{l}{ Total (no 203) } & Ativos ( $\mathrm{n}$ - 123) & \multicolumn{2}{l}{ Inativos (no 80) } & $\mathrm{p}$ \\
\cline { 2 - 5 } & Média dp & Média dp & Média dp & $\mathrm{d}$ \\
\hline Acessibilidade (0 a 18) & $8,39 \pm 4,664$ & $8,22 \pm 4,403$ & $8,66 \pm 5,057$ & 0,682 \\
Calçadas (0 a 1) & $0,84 \pm 0,365$ & $0,82 \pm 0,385$ & $0,65 \pm 0,478$ & 0,305 \\
Áreas verdes (0 a 2) & $0,75 \pm 0,814$ & $0,76 \pm 0,840$ & $0,95 \pm 0,227$ & 0,637 \\
Poluição geral* (3 a 0) & $0,62 \pm 0,758$ & $0,63 \pm 0,783$ & $0,60 \pm 0,722$ & 0,946 \\
Segurança de trânsito (0 a 3) & $1,62 \pm 0,873$ & $1,59 \pm 0,828$ & $1,65 \pm 0,943$ & 0,548 \\
Segurança geral (0 a 3) & $1,82 \pm 0,905$ & $1,93 \pm 0,885$ & $1,65 \pm 0,915$ & $\mathbf{0 , 0 3 5}$ \\
Apoio Social (0 a 3) & $0,86 \pm 0,934$ & $0,97 \pm 0,966$ & $0,70 \pm 0,863$ & $\mathbf{0 , 0 4 6}$ \\
Animal & $0,75 \pm 0,814$ & $0,76 \pm 0,840$ & $0,73 \pm 0,775$ & 0,942 \\
\hline
\end{tabular}

*Somatório invertido: quanto mais próximo de 'zero' melhor o resultado para a atividade física.

**Amostra considerada foi das pessoas que tinham animais de estimação.

No que diz respeito a Lajeado, a amostra do estudo foi composta por 203 indivíduos entre homens e mulheres. Destes, $21,7 \%$ foram considerados fisicamente ativos intensos, 38,9\% fisicamente ativos 
moderados e 39,4\% sedentários. Ao separarmos a amostra em ativos e sedentários (tabela 4), obtemos 60,6\% de ativos e 39,4\% de sedentários. A percepção do ambiente em relação ao nível de atividade física trouxe como variáveis que tiveram valor de significância em favor dos ativos fisicamente os escores de segurança geral $(p<0,035)$ e de apoio social $(p<0,046)$.

\section{DISCUSSÃO}

O escore geral de acessibilidade e conveniência em Muçum, Roca Sales e Lajeado mostraram valores de 8,25; 7,41 e 8,39, respectivamente, escores inferiores ao estudo em Ermelino Matarazzo, entre 14,60 em um máximo de 18 (FLORINDO et al., 2012), que possuía densidade demográfica substancialmente maior na relação com os municípios do interior gaúcho, tendo 15.419 hab./km² contra 43,20; 49,29 e 793,07 hab./km². Características das regiões, como a densidade populacional, tem forte ligação com a percepção do ambiente para a prática AF. Os números sugerem que a densidade favorece a acessibilidade a pontos de interesse, o que promove melhores níveis de AF. Indivíduos que residem em áreas mais densas e com melhor conexão de ruas e uso do solo mais diversificado tendem a ser mais ativos, apesar de concentrar um maior número de veículos automotores, entre outros possíveis complicadores para um deslocamento ativo (FRANK et al., 2006; OWEN et al., 2007; DYCK et al., 2010; ADAMS et al., 2011). Ainda, estudo de Hino et al. (2017) sustenta que a prática física está associada ao acesso a espaços públicos, assim como à estética do ambiente, firmando que quanto mais acessível são os pontos de interesse das pessoas, maior a possibilidades de ter-se uma população mais ativa fisicamente. Relevante apontar que, mesmo Lajeado tendo uma densidade bastante superior aos outros municípios estudados e conservar características de centros maiores, como o percentual de população residindo em área urbana, ainda não reúne características para um aumento da acessibilidade e conveniência.

O estudo de Hino et al. (2010), na cidade de Curitiba-PR, auxilia a sustentar a relação entre AF e densidade, constatando que pessoas que residem em áreas de maior renda, com maior número de academias e próximos a centros de esporte e lazer, tendem a caminhar mais quando comparadas com pessoas que moram em áreas sem estas características de acesso e, ainda, menos populosas, onde a distância faz da AF uma questão menos atrativa como forma de viagem. É importante apontar que Muçum e Roca Sales se caracterizam por serem cidades de pequeno porte com extensa área, quando em relação ao número de habitantes e zona urbana mais dispersa. Da mesma forma, Lajeado, apesar de ter densidade consideravelmente maior do que seus pares no interior gaúcho e estar entre uma das dez cidades com maior densidade do RS, ainda difere consideravelmente da região do distrito paulista.

No que tange ao aspecto segurança geral, no presente estudo, apenas Lajeado apresentou diferença significativa em favor dos ativos. Nos dois municípios ainda menores, tanto em adultos quanto em idosos, não houve escores diferentes entre ativos e sedentários, assim como no estudo de Lim et al. (2005) com 8.881 idosos em New South Wales, na Austrália. Apesar das distinções entre a região australiana e os municípios gaúchos, o contexto de segurança pode sofrer influência das características dos municípios de interior, no caso Muçum e Roca Sales, que habitualmente são mais pacatos e têm índices de criminalidade 
condizentes com sua menor população e características específicas. No que diz respeito à cidade australiana, a condição de país desenvolvido pode ser o diferencial para a não associação deste escore. Já o estudo de Pereira et al. (2019), obteve resultados significativos relativos à percepção de segurança para caminhar, andar de bicicleta ou praticar esportes perto de casa durante a noite no município de Canindé, cidade de pequeno porte do Ceará. No que tange a Lajeado, entende-se que, apesar de ainda ser identificado como sendo um município pequeno, o mesmo apresenta características de cidades maiores, com economia mais ativa, com movimento de maiores recursos e maior urbanização. Nos estudos de Florindo et al. (2011) com 890 adultos no distrito de São Paulo, de Wood et al. (2008) com 335 e de Giles-Corti et al. (2002) com 1.803 adultos australianos, foi constatado que as pessoas mais ativas no deslocamento tinham uma percepção positiva de segurança do local onde residiam. No que tange a idosos, autores como Salvador et al. (2009) sugerem que indivíduos que residem em regiões de baixa renda ou com maiores desigualdades sociais, tendem a apresentar menores níveis de atividade física, seja no lazer como também no deslocamento, devido ao sentimento de insegurança. Ainda em relação à criminalidade, em cálculo da divisão entre o número de ocorrências criminais divididas pelo número de habitantes, Roca Sales e Muçum apresentam o índice de 0,01 crimes por habitante. Em Lajeado esse índice sobre para 0,02. Já em Porto Alegre, capital do estado e grande centro urbano, esse número chega a 0,05 (SSPRS, 2019).

No que diz respeito à segurança no trânsito, em que a percepção de maior segurança decorre de melhores condições envolvendo respeito de motoristas e presença de faixas de segurança, por exemplo, houve diferença significativa para a AF apenas na cidade de Muçum. Em Roca Sales a não associação pode ter relação à condição dos idosos de menor exposição ao trânsito, o que também se ampara pelos níveis de atividade física terem relação ao trabalho doméstico, e por isso não perceberem este segmento em seu diaa-dia. Este resultado vai de encontro aos achados de Balbé et al. (2018) em Rio do Sul-SC e de Barnett et al. (2017), no qual a segurança de trânsito, assim como o acesso a espaços para a prática física, a densidade e a estética, estão associados a melhores níveis de AF. Lajeado também não apresenta esta diferença entre ativos e inativos, assim como nos estudos anteriores nas cidades de Curitiba e em Ermelino Matarazzo (HINO et al., 2010; FLORINDO et al., 2011). O volume do trânsito das pequenas cidades tende a ser menor, mas existe diferença relevante entre os mesmos pois a margem populacional e de urbanização que os difere é muito ampla. Exemplo são as cidades foco deste estudo, em que duas apresentam populações de menos de 15 mil habitantes e a terceira está próximo dos 85 mil habitantes.

O escore de calçadas apresentou relação positiva apenas no estudo com os idosos em Roca Sales assim como nas duas grandes cidades brasileiras - Curitiba e São Paulo - e diferente de Muçum e Lajeado. Florindo et al. (2013) indicam que calçadas estão associadas com a prática AF. Para tanto, cidades menores, pela própria área e densidade e ao menos sob percepção de idosos, tendem a ter melhor conservado o passeio público nas áreas urbanas, regiões onde os estudos foram realizados, favorecendo o deslocamento ativo.

O escore de apoio social, ou seja, o convite de amigos ou familiares ou ainda as oportunidades para a prática de atividades física nas proximidades da residência apresentaram associação positiva significativa 
com a AF dos adultos nas cidades de Muçum e Lajeado a partir de uma maior motivação e envolvimento social. Os dados corroboram com os estudos de Reis et al. (2011) e Bourdeaudhuij et al. (2005). Ademais, Treiber et al. (1991) também encontraram diferenças entre a fonte de apoio e diferentes tipos de AF. Ainda com diferença significativa, o escore animal de estimação apresentou significância na cidade de Muçum. Adultos que tinham cães e os levavam para passear tiveram maiores chances de caminhar no deslocamento, apresentando melhores níveis de AF. O resultado vai ao encontro das pesquisas de Clarke et al. (2009), assim como com os estudos de Giehl et al. (2012), que sugerem ainda que ter cães e realizar passeios com os mesmos pode, para além da AF, promover uma maior interação social.

Por fim, é relevante o percentual de ativos fisicamente entre os idosos de Roca Sales, onde a maior parte do escore resulta de atividades realizadas em casa, se associando ao estudo de Chaudhury et al. (2016). O presente resultado encontra explicação na observação das características regionais, onde o escore clima pode estar implicado na ação da prática física, não identificou diferença significativa entre sedentários e ativos. Os resultados evidenciam características de idosos da região estudada, ou seja, de uma vida mais caseira e trabalho doméstico por conta de fatores climáticos com outono e inverno chuvosos e frios, além de características próprias do município em termos de ofertas para a prática física, entre outros. Idosos tendem a se expor menos às intempéries características da região sul do Brasil. O trabalho doméstico, neste caso, aumenta os níveis de AF sem ter a influência do clima, diferente do que aponta Tucker et al. (2007), para quem o clima é um dificultador para melhores níveis de AF de forma geral. A possível menor exposição acaba por não influenciar a não associação do clima com AF a partir da percepção do ambiente neste estudo. $\mathrm{O}$ alto percentual de ativos fisicamente entre os idosos de Roca Sales contrapõe achados de Sallis et al. (2016), para quem os idosos não atingem à necessidade diária de AF e também de Pereira et al. (2019), que apontam em seu estudo que apenas $41 \%$ da população idosa é ativa fisicamente.

\section{CONCLUSÕES}

Os escores de acessibilidade, segurança de trânsito, apoio social e ter animal de estimação, apresentaram diferença significativa em prol dos ativos fisicamente no que diz respeito aos adultos de Muçum. Para os idosos de Roca Sales, o único escore que apresentou esta condição foi o que se refere à qualidade de calçadas. Já na cidade de Lajeado, o escore apoio social foi o único que apresentou diferença significativa em prol dos adultos ativos fisicamente. Os demais escores como segurança geral, poluição, topografia de ruas e clima não apresentaram diferenças entre ativos e inativos fisicamente, nem entre adultos e nem entre idosos.

A análise da relação entre ambiente e AF sugere observação atenta das características das localidades. Municípios menores podem ter maiores distâncias entre residências e pontos de interesse por conta da relação de oferta de serviços em resposta à demanda de população. Mesmo em cidades que reúnem características mais próximas de maiores centros por conta da urbanização e densidade, por exemplo, ainda não reúnem condições para fazer da densidade um fator positivo para a prática de AF. No entanto, outras características, conferidas pelo ambiente, como segurança, trânsito, clima e níveis de poluição podem 
interferir nestes números.

Ainda, percebe-se que ao mesmo tempo em que a maior densidade se alinha a um caminhar ativo por conta da acessibilidade e conveniência, também favorece a um número maior de veículos motorizados por conta do próprio número de pessoas, acarretando um maior nível de emissões atmosféricas. De outra parte, pela menor acessibilidade e conveniência em municípios menores, onde as distâncias seguem uma lógica inversamente proporcional, a população tende a ser menos ativa no deslocamento, indicando que um melhor planejamento dos espaços urbanos influencia na relação dos cidadãos com o ambiente. Melhor segurança e qualidade do passeio público favorecem o transporte ativo e a redução de veículos, contribuindo não somente com a relação dos cidadãos com o ambiente, mas também com o bem-estar das pessoas e com a qualidade ambiental.

\section{REFERÊNCIAS}

BOURDEAUDHUIJ, I.; TEIXEIRA, P. J.; CARDON, G.; DEFORCHE, B.. Environmental and psychosocial correlates of physical activity in Portuguese and Belgian adults. Public Health Nutrition, v.8, n.7, p.886-895, 2005. DOI: https://doi.org/10.1079/phn2005735

ANDRADE, C. E. S.; D'AGOSTO, M. A.. Avaliação dos sistemas metroviários nas emissões de $\mathrm{CO}_{2}$ : análise comparativa das emissões por automóveis, ônibus e metrôs. Engenharia Sanitária e Ambiental, Rio de Janeiro, v.24, n.5, p.919-927, 2019. DOI: https://doi.org/10.1590/s1413-41522019139710

ARANGO, C. M.; PÁEZ, D. C.; REIS, R. S.; BROWNSON, R. C.; PARRA, D. C.. Associação entre ambiente percebido e atividade física entre adultos na América Latina: uma revisão sistemática. International Journal of Behavioral Nutrition and Physical Activity. v.10, n.122, 2013. DOI: https://doi.org/10.1186/1479-5868-10-122

BAKICI, T.; ALMIRALL E.; WAREHAM, J.. A Smart City Initiative: The Case of Barcelona. Journal of the Knowledge Economy, v.2, n.1, p.1-14, 2012. DOI: https://doi.org/10.1007/s13132-012-0084-9

BALBÉ, G. P.; BIESDORF, M.; SOUZA, J. C.; SANTOS, L. C.; SCHLEM, C. D. S.; WATHIER, C. A.. O contexto do ambiente percebido na atividade física de lazer e deslocamento em idosos. Licere, Belo Horizonte, v.21, n.2, 2018. DOI: https://doi.org/10.35699/1981-3171.2018.1814

BARNETT, D. W.; BARNETT, A.; NATHAN, A.; CAUWENBERG, J. V.; CERIN, E.. Built environmental correlates of older adults' total physical activity and walking: a systematic review and meta-analysis. International Journal of Behavioral Nutrition and Physical Activity, v.14, n.103, 2017. DOI: https://doi.org/10.1186/s12966-017-0558-z

BROWNSON, R. C.; HOEHNER, C. M.; DAY, K.; FORSYTH, A.; SALLIS, J. F.. Measuring the built environment for physical activity: state of the science. American Journal of Preventive Medicine, v.36, n.4, p.99-123, 2009. DOI: https://doi.org/10.1016/j.amepre.2009.01.005

CHAUDHURY, H.; CAMPO, M.; MICHAEL, Y.; MAHMOOD, A.. Neighbourhood environment and physical activity in older adults. Social Science \& Medicine, v.149, p.104-113, 2016.
DOI: https://doi.org/10.1016/j.socscimed.2015.12.011

CLARKE, P.; AILSHIRE, J. A. LANTZ, P.. Urban built environments and trajectories of mobility disability: Findings from a national sample of community-dwelling American adults (1986-2001). Social Science \& Medicine, v.69, n.6, p.964-970, 2009. DOI: https://doi.org/10.1016/j.socscimed.2009.06.041

CNT. Confederação Nacional do Transporte. Oficina Nacional: transporte e mudança climática. Brasília: CNT, 2009.

CARVALHO, C. H. R.. Emissões relativas de poluentes do transporte motorizado de passageiros nos grandes centros urbanos brasileiros. Texto para Discussão. IPEA, 2011.

DANISH; ZHANG, B.; WANG, B.; WANG, Z.. Role of renewable energy and non-renewable energy consumption on EKC: Evidence from Pakistan. Journal of Cleaner Production, v.156, p.855-864, 2017. DOI: https://doi.org/10.1016/j.jclepro.2017.03.203

DYCK, D. V.; CARDON, G.; DEFORCHE, B.; SALLIS, J. F.; OWEN, N.; DE BOURDEAUDHUIJ, I.. Neighborhood SES and walkability are related to physical activity behavior in Belgian adults. American Journal of Preventive Medicine, v.50, n.1, p.74-79, 2010. DOI:

https://doi.org/10.1016/j.ypmed.2009.07.027

FLORINDO, A. A.; SALVADOR, E. P.; REIS, R. S.; GUIMARÃES, V. V.. Percepção do ambiente e prática de atividade física em adultos residentes em região de baixo nível socioeconômico. Revista Saúde Pública, v. 45, n. 2, p. 302-310, 2011. DOI: https://doi.org/10.1590/\$0034-89102011000200009

FLORINDO A. A.; GUIMARÃES, V. V.; FARIAS JUNIOR, J. C; SALVADOR, E. P.; SÁ, T. H.; REIS, R. S.; HALLAL, P. C.. Validação de uma escala de percepção do ambiente para a prática de atividade física em adultos de uma região de baixo nível socioeconômico. Revista Brasileira de Cineantropometria e Desenvolvimento Humano, São Paulo, v.14, n.6, 2012. DOI: http://dx.doi.org/10.5007/1980$\underline{0037.2012 \mathrm{v} 14 \mathrm{n} 6 \mathrm{p} 647}$

FLORINDO, A. A.; GARCIA, L. M. T.; GUIMARÃES, V. V.; 
SALVADOR, E. P.; FILHO, H. F.; REIS, R. S.; FARIAS JUNIOR, J. C.. Escore de ambiente construído relacionado com a prática de atividade física no lazer: aplicação numa região de baixo nível socioeconômico. Revista Brasileira de

Cineantropometria e Desenvolvimento Humano, v.15, n.2, p.243-255, 2013. DOI: https://doi.org/10.5007/1980$\underline{0037.2013 v 15 n 2 p 243}$

FRANK, L. D.; ENGELKE, P.. Multiple impacts of the built environment on public health: Walkable places and the exposure to air pollution. International Regional Science Review, v.28, n.2, p.193-216, 2005. DOI: https://doi.org/10.1177/0160017604273853

FRANK L. D.; CONWAY, T. L.; SALLIS, J. F.; CHAPMAN, J. Many Pathways from Land Use to Health: Associations between Neighborhood Walk-ability and Active Transportation, Body Mass Index, and Air Quality. Journal of the American Planning Association, v.72, n.1, p.75-87, 2006. DOI: https://doi.org/10.1080/01944360608976725

GIEHL, M. W. C.; SCHNEIDER, I. J. C.; CORSEUIL, H. X.; BENEDETTI, T. R. B.; D'ORSI, E.. Physical activity and environment perception among older adults: a population study in Florianópolis, Brazil. Revista Saúde Pública, v.46, n.3, p.516-525, 2012. DOI: https://doi.org/10.1590/S0034$\underline{89102012005000026}$

GILES-CORTI, B.; DONOVAN, R. J.. Socioeconomic Status Differences in Recreational Physical Activity Levels and Real and Perceived Access to a Supportive Physical Environment. American Journal of Preventive Medicine, v.35, n.6, p.601611, 2002. DOI: https://doi.org/10.1006/pmed.2002.1115

HANDY, S.; BOARNET, M. G.; EWING, R.; KILLINGSWORTH, R. E.. How the built environment affects physical activity. Views from urban planning. American Journal of Preventive Medicine, v.23, n.2S, p.64-73, 2002. DOI: https://doi.org/10.1016/s0749-3797(02)00475-0

HINO, A. A. F.; REIS, R. S.; FLORINDO, A. A.. Ambiente construído e atividade física: uma breve revisão dos métodos de avaliação. Revista Brasileira de Cineantropometria e Desenvolvimento Humano, Florianópolis, v.12, n.5, p.387-394, 2010. DOI: https://doi.org/10.5007/1980-0037.2010V12N5P387

HINO, A. A. F.; RECH, C. R.; GONÇALVES, P. B.; REIS, R. S.. Ambiente percebido do bairro e atividade física no lazer em adultos de Curitiba, Brasil. Revista Brasileira de Cineantropometria e Desenvolvimento Humano, Florianópolis, v.19, n.5, p.596-607, 2017. DOI: https://doi.org/10.5007/1980-0037.2017v19n5p596

LEITE, C.; AWAD, J. C. M.. Cidades sustentáveis, cidades inteligentes: desenvolvimento sustentável num planeta urbano. Porto Alegre: Bookman, 2012.

LIM, K.; TAYLOR, L.. Factors associated with physical activity among older people - a population based study. American Journal of Preventive Medicine, v.40, n.1, p.33-40, 2005. DOI: https://doi.org/10.1016/j.ypmed.2004.04.046

MALAVASI, L. M.; DUARTE, M. F. S.; BOTH, J.; REIS, R. S.. Escala de mobilidade ativa no ambiente comunitário - news Brasil: retradução e reprodutibilidade. Revista Brasileira de Cineantropometria e Desenvolvimento Humano, v.9, n.4, p.339-350, 2007.

MATSUDO, S.; ARAÚJO, T.; MATSUDO, V.; ANDRADE, D.; ANDRADE, E.; OLIVEIRA, L. C.; BRAGGION, G.. Questionário internacional de atividade física (I PAQ): estudo de valldade e reprodutibilidade no brasil. Revista Atividade física e Saúde, v.6, n.2, p.5-18, 2001. DOI: https://doi.org/10.12820/rbafs.v.6n2p5-18

OWEN, N.; CERIN, E.; LESLIE, E.; DUTOIT, L.; COFFEE, N.; FRANK, L. D.; BAUMAN, A. E.; HUGO, G.; SAELENS, B. E.; SALLIS, J. F.. Neighborhood walkability and the walking behavior of Australian adults. American Journal of Preventive Medicine, v.33, n.5, p.387-95, 2007. DOI: https://doi.org/10.1016/i.amepre.2007.07.025

PÉLLICO NETTO, S. P.; KAUANO, E. E.; CORAIOLA, M.; WEBER, S. H.; ERDELYI, S.. Estimativa do potencial de neutralização de dióxido de carbono no programa VIVAT Neutracarbono em Tijucas do Sul, Agudos do Sul e São José dos Pinhais, PR. Revista Acadêmica Ciências Agrárias e Ambientais, v.6, n.2, p.293-306, 2008.

PEREIRA, D. S.; SILVA, C. A. B.; SILVA, J. R.; MOREIRA, T. M. M.. Percepção de ambiente e nível de atividade física em idosos do nordeste brasileiro. Coleção Pesquisa em Educação Física, Várzea Paulista, v.18, n.3, p.83-91, 2019.

REIS, M. S.; REIS, R. S.; HALLAL, P. C.. Validity and reliability of a physical activity social support assessment scale. Rev Saúde Pública v.45, n.2, p.294-301, 2011. DOI: https://doi.org/10.1590/s0034-89102011000200008

SALLIS, J. F.; BULL, F.; GUTHOLD, R.; HEATH, G. W.; INOUE, S.; KELLY, P.; OYEYEMI, A. L.; PEREZ, L. G.; RICHARDS, J.; HALLAL, P. C.. Progress in physical activity over the Olympic quadrennium. The Lancet, v.388, n.10051, p.1325-1336, 2016. DOI: https://doi.org/10.1016/S0140-6736(16)30581-5

SALVADOR, E. P.; FLORINDO, A. A.; REIS, R. S.;COSTA, E. F.. Perception of the environment and leisure-time physical activity in the elderly. Revista Saúde Pública, v.43, n.6, p.972-80, 2009. DOI: https://doi.org/10.1590/S003489102009005000082

SOARES, T. C.; CUNHA, D. A.. Emissões de gases de efeito estufa e eficiência ambiental no Brasil. Nova Economia, v.29, n.2, p.429-458, 2019. DOI: http://dx.doi.org/10.1590/0103$\underline{6351 / 3795}$

SSPRS. Secretaria de Segurança Pública do Rio Grande Do Sul. Indicadores criminais. Porto Alegre: SSPRS, 2019.

TUCKER, P.; GILLILAND, J.. The effect of season and weather on physical activity: a systematic review. Public health, v.121, n.12, p.909-922, 2007. DOI: https://doi.org/10.1016/i.puhe.2007.04.009

TREIBER, F. A.; BARANOWSKI, T.; BRADEN, D. S.; STRONG, W. B.; LEVY, M.; KNOX, W.. Social support for exercise: relationship to physical activity in young adults. American Journal of Preventive Medicine, v.20, n.6, p.737-50, 1991. DOI: https://doi.org/10.1016/0091-7435(91)90068-f

WEN, C. P.; WU, X.. Stressing harms of physical inactivity to promote exercise. The Lancet, v.380, p.192-193, 2012. DOI: https://doi.org/10.1016/S0140-6736(12)60954-4 
WOOD, L.; SHANNON, T.; BULSARA, M.; PIKORA, T.; MCCORMACK, G.; GILES-CORTI, B.. The anatomy of the safe and social suburb: an exploratory study of the built environment, social capital and residents' perceptions of safety. Health \& Place, v.14, n.1, p.15-31, 2008. DOI: http://doi.org/10.1016/j.healthplace.2007.04.004

ZHANG, J.; XING, Z.; WANG, J.. Analysis of CO2 emission performance and abatement potential for municipal industrial sectors in Jiangsu, China. Sustainability, v.8, n.7, p.697-711, 2016. DOI: https://doi.org/10.3390/su8070697

A CBPC - Companhia Brasileira de Produção Científica (CNPJ: 11.221.422/0001-03) detém os direitos materiais desta publicação. Os direitos referem-se à publicação do trabalho em qualquer parte do mundo, incluindo os direitos às renovações, expansões e disseminações da contribuição, bem como outros direitos subsidiários. Todos os trabalhos publicados eletronicamente poderão posteriormente ser publicados em coletâneas impressas sob coordenação da Sustenere Publishing, da Companhia Brasileira de Produção Científica e seus parceiros autorizados. Os (as) autores (as) preservam os direitos autorais, mas não têm permissão para a publicação da contribuição em outro meio, impresso ou digital, em português ou em tradução. 